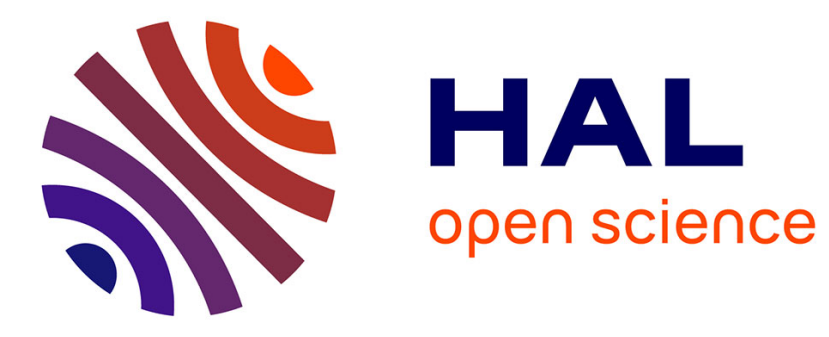

\title{
Contextual Bandit with Adaptive Feature Extraction
} Baihan Lin, Djallel Bouneffouf, Guillermo A Cecchi, Irina Rish

\section{To cite this version:}

Baihan Lin, Djallel Bouneffouf, Guillermo A Cecchi, Irina Rish. Contextual Bandit with Adaptive Feature Extraction. 2018 IEEE International Conference on Data Mining Workshops (ICDMW), Nov 2018, Singapore, Singapore. pp.937-944, 10.1109/ICDMW.2018.00136 . hal-02949763

\section{HAL Id: hal-02949763 https://hal.science/hal-02949763}

Submitted on 26 Sep 2020

HAL is a multi-disciplinary open access archive for the deposit and dissemination of scientific research documents, whether they are published or not. The documents may come from teaching and research institutions in France or abroad, or from public or private research centers.
L'archive ouverte pluridisciplinaire HAL, est destinée au dépôt et à la diffusion de documents scientifiques de niveau recherche, publiés ou non, émanant des établissements d'enseignement et de recherche français ou étrangers, des laboratoires publics ou privés. 


\section{Contextual Bandit with Adaptive Feature Extraction}

\author{
$1^{\text {st }}$ Baihan Lin \\ Columbia University \\ New York, NY, USA \\ baihan.lin@columbia.edu
}

\author{
$2^{\text {nd }}$ Djallel Bouneffouf, Guillermo A. Cecchi, Irina Rish \\ IBM Thomas J. Watson Research Center \\ Yorktown Heights, NY, USA \\ \{dbouneffouf, gcecchi, rish\}@us.ibm.com
}

\begin{abstract}
We consider an online decision making setting known as contextual bandit problem, and propose an approach for improving contextual bandit performance by using an adaptive feature extraction (representation learning) based on online clustering. Our approach starts with an off-line pre-training on unlabeled history of contexts (which can be exploited by our approach, but not by the standard contextual bandit), followed by an online selection and adaptation of encoders. Specifically, given an input sample (context), the proposed approach selects the most appropriate encoding function to extract a feature vector which becomes an input for a contextual bandit, and updates both the bandit and the encoding function based on the context and on the feedback (reward). Our experiments on a variety of datasets, and both in stationary and non-stationary environments of several kinds demonstrate clear advantages of the proposed adaptive representation learning over the standard contextual bandit based on "raw" input contexts. 1
\end{abstract}

Index Terms-multi-arm bandit, contextual bandit, online learning, autoencoder, representation learning, online clustering

\section{INTRODUCTION}

Sequential decision making is a common problem in many practical applications where the agent must choose the best action to perform at each iteration in order to maximize the cumulative reward over some period of time. One of the key challenges is achieving a good trade-off between the exploration of new actions and the exploitation of known actions. This exploration vs. exploitation trade-off in sequential decision making problems is often formulated as the multi-armed bandit (MAB) problem: given a set of bandit "arms" (actions), each associated with a fixed but unknown reward probability distribution [Auer et al., 2002a; Lai and Robbins, 1985], an agent selects an arm to play at each iteration, and receives a reward, drawn according to the selected arm's distribution, independently from the previous actions.

A particularly useful version of MAB is the contextual multiarmed bandit (CMAB), or simply the contextual bandit problem, where at each iteration, before choosing an arm, the agent observes an $N$-dimensional context, or feature vector. Over time, the goal is to learn the relationship between the context vectors and the rewards, in order to make better prediction which action to choose given the context [Agrawal and Goyal 2013], as an important problem setting in reinforcement learning [Bouneffouf et al. 2017a; Lin et al. 2019, 2020a b b ]

${ }^{1}$ The data and codes to reproduce all empirical results can be accessed at https://github.com/doerlbh/ABaCoDE
For example, the contextual bandit approach is commonly used in various practical sequential decision problems with side information (context), from clinical trials [Villar et al., 2015], to speaker recognition systems [Lin and Zhang, 2020a b], to recommender system [Mary et al. [2015], where the patient's information (medical history, etc.), or a speaker's voice profile, or an online user's purchase profile provide a context for making a better decision about a potential treatment or a speaker identity or an ad to show, and the reward represents the outcome of the selected action, such as, for example, success or failure of a particular treatment option.

However, in certain real-life applications, before the online decision-making starts, an agent may have an access to a unlabeled context history (i.e., contexts without the associated rewards), which can be potentially used as a prior knowledge to improve the subsequent online decision-making. For instance, in medical decision-making settings, the doctor may have an access to medical records of different patients, which can be used to gain a better understanding of the patients population. A different example of unlabeled context history can occur in an online recommender setting, where the system may have some previous information about the users, although the reward feedback (e.g., whether the user clicked on the suggested link or not) might be missing.

Having an access to unlabeled data makes it possible to pretrain some model of the input (contexts) in an offline mode, and use it later to improve the online decision making. For example, we can learn an autoencoder to map the raw inputs into potentially better representations. Moreover, when the inputs are non-homogeneous, we may want to cluster the unlabeled data and learn separate representations for each cluster. Then, in the online mode, we can decide which representation to use for a given context; such context-driven representation selection has a potential to further improve the subsequent decisionmaking. These representation models (e.g., autoencoders) can (and should) continue to be updated online as more contexts become available, especially in nonstationary environments abundant in practical applications, where both the context and reward distributions can change in various ways.

Motivated by the above scenarios, we consider here a contextual bandit setting, called Contextual Bandit with Representation learning and unlabeled History $(\mathrm{CBRH})$. In this setting, first of all it is assumed that (1) there is some set of unlabeled contexts available for pre-training, before the online decisionmaking starts, which allows for an initial clustering and encoder 
construction; (2) the bandit's performance can be improved by learning a good context representation (embedding) rather than using the raw input, the (3) embedding functions are pre-trained on the unlabeled history and adaptively selected (and updated) based on the context during the online decision-making. Next, we propose an algorithm for the above CBRH setting, called Adaptive Bandit with Context-Driven Embeddings ( $A B a C o D E)$, which implements online, clustering-based encoding selection and learning coupled with Thompson-Sampling approach.

We evaluate our approach on several types of nonstationary environments and demonstrated that (1) using embeddings, in general, considerably improves performance of contextual bandit; and (2) moreover, in several cases, adaptive, contextdependent type of embeddings are much better than just one, "uniform" embedding.

Overall, the lesson learned is that the embedding based approach propose here can be a useful tool for improving the performance of contextual bandit; it is helpful to have an access to some "unlabeled" history of contexts to create a reasonable initial embeddings to start with, and to keep augmenting them with respect to new instances arriving in online mode.

To summarize, our approach has several advantages over the standard contextual bandit: it can exploit the unlabeled context history to learn useful context representations; it allows for a flexible, adaptive online selection of context-specific representations, as well as for continuous learning/adaptation of such representations.

\section{RELATED WORK}

The multi-armed bandit problem has been extensively studied. Optimal solutions have been provided using a stochastic formulation [Auer et al., 2002a; Lai and Robbins, 1985], a Bayesian formulation [Agrawal and Goyal, 2012, Bouneffouf and Féraud, 2016, Thompson, 1933], or using an adversarial formulation [Auer and Cesa-Bianchi, 1998, Auer et al. 2002b]. However, these approaches do not take into account the context which may affect to the arm's performance. In LINUCB [Chu et al., 2011; Li et al. 2010] and in Contextual Thompson Sampling style (CTS) algorithms [Agrawal and Goyal, 2013 Bouneffouf et al. [2017b], the authors assume a linear dependency between the expected reward of an action and its context; the representation space is modeled using a set of linear predictors. This assumption is not used in Neural Bandit [Allesiardo et al., 2014]. However, these algorithms assume that the agent can observe the reward at each iteration, which is not the case in many practical applications, including those discussed earlier in this paper.

Authors in [Bartók et al. [2014] studies considering some kind of incomplete feedback called "Partial Monitoring (PM)", which is a general framework for sequential decision making problems with incomplete feedback that allows the learner, when it is possible, to retrieve the expected value of actions through an analysis of the feedback matrix, both of which are assumed to be known to the learner.

In [Gajane et al. [2016] authors study a variant of the stochastic multi-armed bandit (MAB) problem in which the rewards are corrupted. In this framework, motivated by privacy preserving in online recommender systems, the goal is to maximize the sum of the (unobserved) rewards, based on the observation of transformation of these rewards through a stochastic corruption process with known parameters.

We can say that our setting is similar to the online semisupervised learning [Lin, 2020b; Ororbia et al., 2015, Yver. 2009], which is a field of machine learning that studies learning from both labeled and unlabeled examples in an on-line setting. However, in their setting the true label is received at each iteration, while in our setting a bandit feedback is assumed, i.e., if classification was incorrect, the agent will not know what the correct label was, only that its decision was incorrect.

\section{BACKGROUND}

This section introduces some background concepts our approach builds upon, such as contextual bandit and Thompson Sampling.

\section{The contextual bandit problem}

Following [Langford and Zhang |2008], this problem is defined as follows. At each time point (iteration) $t \in\{1, \ldots, T\}$, an agent is presented with a context (feature vector) $\mathbf{x}_{t} \in \mathbf{R}^{N}$ before choosing an arm $k \in A=\{1, \ldots, K\}$. We will denote by $X=\left\{X_{1}, \ldots, X_{N}\right\}$ the set of features (variables) defining the context. Let $\mathbf{r}_{t}=\left(r_{t}^{1}, \ldots, r_{t}^{K}\right)$ denote a reward vector, where $r_{t}^{k} \in[0,1]$ is a reward at time $t$ associated with the arm $k \in A$. Herein, we will primarily focus on the Bernoulli bandit with binary reward, i.e. $r_{t}^{k} \in\{0,1\}$. Let $\pi: X \rightarrow A$ denote a policy. Also, $D_{c, r}$ denotes a joint distribution over $(\mathbf{x}, \mathbf{r})$. We will assume that the expected reward is a linear function of the context, i.e. $E\left[r_{t}^{k} \mid \mathbf{x}_{t}\right]=\mu_{k}^{T} \mathbf{x}_{t}$, where $\mu_{k}$ is an unknown weight vector (to be learned from the data) associated with the arm $k$.

\section{Contextual Thompson Sampling}

In this setting, we consider the general Thompson Sampling, where the reward $r_{t}^{i}$ for choosing arm $i$ at time $t$ follows a parametric likelihood function $\operatorname{Pr}\left(r_{t} \mid \tilde{\mu}_{i}\right)$. Following [Agrawal and Goyal, 2013], the posterior distribution at time $t+1$, $\operatorname{Pr}\left(\tilde{\mu}_{i} \mid r_{t}\right) \propto \operatorname{Pr}\left(r_{t} \mid \tilde{\mu}_{i}\right) \operatorname{Pr}\left(\tilde{\mu}_{i}\right)$ is given by a multivariate Gaussian distribution $\mathcal{N}\left(\hat{\mu}_{i}(t+1), v^{2} B_{i}(t+1)^{-1}\right)$, where $B_{i}(t)=I_{d}+\sum_{\tau=1}^{t-1} x_{\tau} x_{\tau}^{\top}$, and where $d$ is the size of the context vectors $\mathbf{x}_{i}, v=R \sqrt{\frac{24}{\epsilon} d \ln \left(\frac{1}{\gamma}\right)}$ with $\left.\left.R>0, \epsilon \in\right] 0,1\right]$, $\gamma \in] 0,1]$ constants, and $\hat{\mu}_{i}(t)=B_{i}(t)^{-1}\left(\sum_{\tau=1}^{t-1} x_{\tau} r_{\tau}\right)$. At every step $t$, the algorithm generates a $d$-dimensional sample $\tilde{\mu}_{i}$ from $\mathcal{N}\left(\hat{\mu}_{i}(t), v^{2} B_{i}(t)^{-1}\right)$, for each arm, selects the arm $i$ that maximizes $x_{t}^{\top} \tilde{\mu}_{i}$, and obtains reward $r_{t}$. 


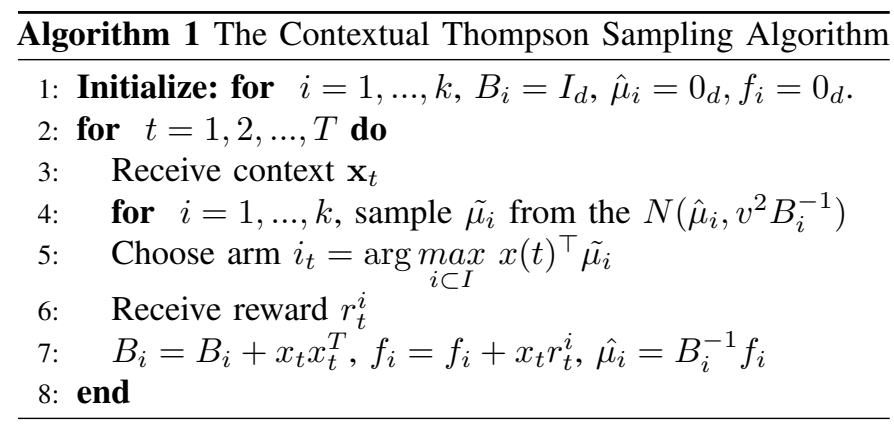

\section{Problem Formulation}

Using the notation introduced in the previous section, we now define our novel bandit setting: Contextual Bandit with Representation learning and unlabeled History $(\mathrm{CBRH})$ (outlined in Alg. 2), based on the following key assumptions.

First, we assume that a context $\mathbf{x}_{t} \in \mathbf{R}^{N}$ is mapped into its representation $\mathbf{z}_{t} \in \mathbf{R}^{N_{i}}$ using an embedding function $e_{i}\left(\mathbf{x}_{t}\right)$, selected from a set $E=\left\{e_{1}, \ldots, e_{k}\right\}$ of currently available embedding functions. Second, we assume that the set of embedding functions $E$ can be modified online. And third, an access to a set $\mathbf{D}$ of unlabeled contexts, i.e. contexts without the associated rewards, is assumed. This dataset can be used, for example, for pre-training embedding functions $e(\mathbf{x})$. We then define a set $\Pi=\cup_{e_{i} \in E}\left\{\pi: \mathbf{R}^{N} \rightarrow A, \pi(\mathbf{x})=\hat{\pi}_{i}\left(e_{i}(\mathbf{x})\right)\right\}$ of compound-function policies, where the function $\hat{\pi}: \mathbf{R}^{N_{i}} \rightarrow A$ maps $\mathbf{z}_{t}=e_{i}\left(\mathbf{x}_{\mathbf{t}}\right)$ to an action in $A$. The objective is to learn a hypothesis $\pi$ over $T$ iterations maximizing the cumulative reward.

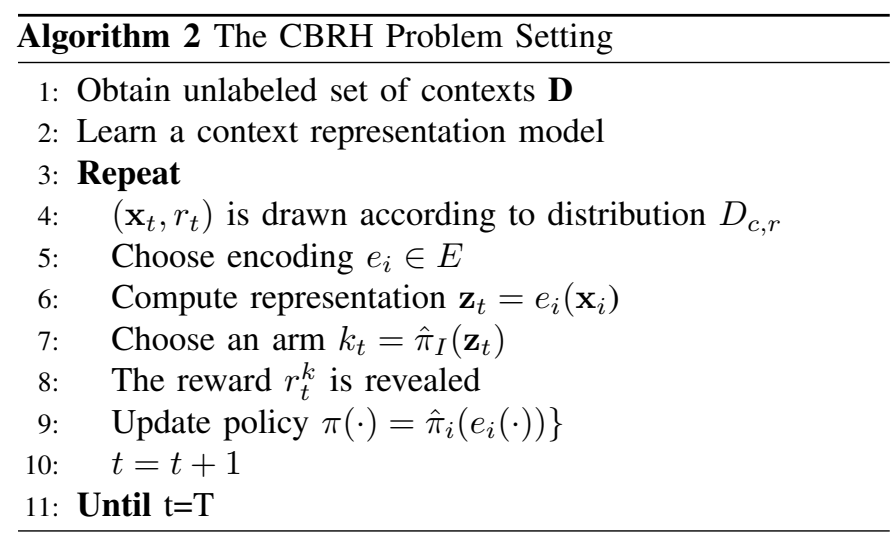

\section{Adaptive Bandit with Context-Dependent EMBEDDINGS (ABACODE)}

We now describe an adaptive, context-driven embedding selection approach to solving the CBRU problem introduced in the previous section. It has two variants, based on onlineand offline clustering, respectively; the choice is controlled by a Boolean input parameter isOnline in Algorithm 3 . Two more inputs include: an unlabeled pre-training dataset $\mathbf{D}$, as well as the number of embeddings $k$. The algorithm processes the input contexts sequentially, one by one, but at the end of each mini-batch of data it updates the embeddings to reflect possible changes in the data distribution.

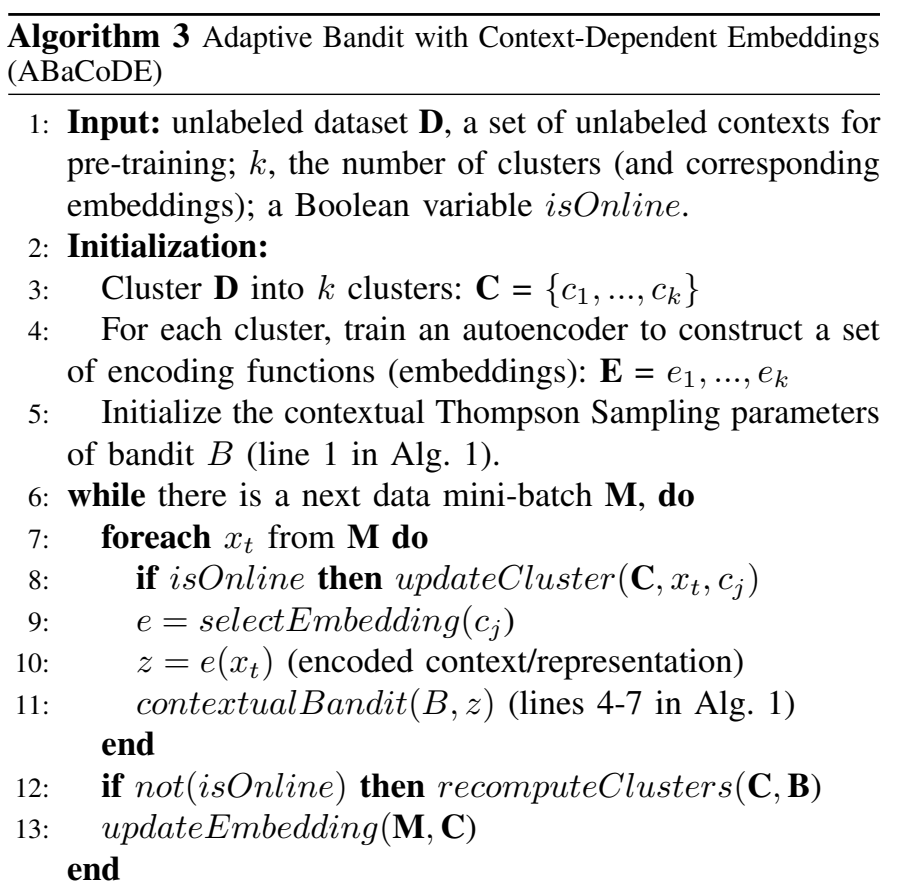

The initialization step consists of clustering the pre-training dataset $\mathbf{D}$ into $k$ clusters (line 3), training an autoencoder for each cluster, which results into $k$ encoding (embedding) functions (line 4), and initializing parameters of the contextual Thompson Sampling bandit, used later to make classification decisions based on embedded context (line 5).

Next, the algorithm switches to the online mode, processing an online stream of incoming samples (contexts). As mentioned above, we assume that at the end of each fixed-length time window, i.e. a fixed-size mini-batch of data, we update our embeddings.

Within each data mini-batch $\mathbf{M}$ (line 7), once the next input sample $\mathbf{x}_{i}$ arrives, it is first assigned to one of the existing clusters $c_{j}$ (line 8), associated with the corresponding embedding function $e_{j}$. Next, an online clustering is performed if isOnline is true, i.e. the centroid of the cluster $c_{j}$ is recomputed, but no changes are made to other clusters (line 9). Otherwise, there are no changes to clusters, until the end of the batch, as we will see shortly. Based on the cluster assignment $c_{j}$, the corresponding embedding function $e_{j}$ is used to compute the representation vector $z$ for given input $\mathbf{x}_{i}$ (line 10); given the context $z$, the contextual bandit $B$ makes a decision (line 11), obtains the reward $r_{i}$ (line 12), and updates its parameters (line 13) using the contextual Thompson Sampling described in the previous section.

After the end of the mini-batch $\mathbf{M}$ is reached (line 14), if isOnline was false, the clusters will be recomputed from scratch using all data points received so far (however, no such re-clustering is performed if the online clustering was selected). Finally, the embeddings (i.e., their corresponding autoencoder parameters) are updated respectively using the updated set of clusters $\mathbf{C}$.

In the next section, we present empirical results comparing 
both online and offline clustering methods outlined above with two baseline approaches:

- Contextual Bandit (CB): as the baseline, we use the standard contextual multi-armed bandit with Thompson Sampling, based on the raw input (i.e., no embeddings).

- universal embedding ( $u E$ ): a universal embedding denotes a single embedding computed based on all data, and always recomputed to include the data from the most recent mini-batch; no clustering is performed.

- mini-batch embedding $(\mathrm{mE})$ : this is our offline clustering approach presented in Algorithm 3, when isOnline is false.

- online embedding $(o E)$ : this is the online version of our algorithm described above, i.e. isOnline is true.

\section{EMPIRICAL EVALUATION}

\section{A. Datasets}

We evaluated our approach on four imaging datasets: MNIST [LeCun, 1998], STL-10 [Coates and Ng, 2011], CIFAR-10 [Coates et al., 2011], Caltech-101 Silhouettes-28 [Griffin et al. 2007] and Warfarin [Consortium and others, 2009] (for details of each dataset, see Table II). To simulate an online data stream, we draw samples from each dataset sequentially, starting from the beginning each time we draw the last sample. At each round, the algorithm receives reward 1 if the instance is classified correctly, and 0 otherwise. We compute the total number of classification errors as a performance metric. However, Warfarin dataset is different, as it was actually produced in a real bandit setting, rather than classification setting.

Bandit vs. classification feedback: important distinction. It is important to keep in mind that the bandit feedback (correct/incorrect classification) makes the classification problem significantly more challenging, as compared to the standard supervised learning, since the true label is never revealed in bandit setting unless the classification is correct. Thus, the classification accuracy in a bandit setting is expected to be lower than in the supervised learning setting - which is not due to inferiority of bandit decision making algorithm versus classifiers, but due to increased problem difficulty, i.s. the lack of feedback about what the correct decision should have been. Recall that such bandit feedback is often a much more realistic model of agent's interaction with the world, especially in online decision making applications such as online advertisement, clinical trials, and so on, which do not fit into classification framework.

However, for empirical evaluation purposes, it is common to use available classification datasets to simulate an online environment with the bandit feedback (i.e., simulating the situation where the bandit receives, for example, 1 or 0 for correct or incorrect decision, but is not told what the correct decision should have been when he receives 0 ; such feedback is different from standard online classification feedback in case of non-binary classification)). We use several classification datasets here for such simulations.

We now describe some details of the experiments. For MNIST, we took 10,000 samples from the original test dataset
TABLE I: Datasets

\begin{tabular}{l|c|c|r|r} 
Datasets & History & Instances & Features & Classes \\
\hline MNIST & 10000 & 20000 & 784 & 10 \\
STL-10 & 20000 & 10000 & 1000 & 10 \\
CIFAR-10 & 2000 & 10000 & 3072 & 10 \\
Caltech-101 S & 671 & 8000 & 784 & 101 \\
Warfarin & 528 & 5000 & 93 & 3 \\
\hline mix: MNIST/Warfarin & 10528 & 10000 & 93 & 13 \\
\hline
\end{tabular}

(clearly, not using them later for testing) to pre-train the encodings, and 60,000 samples from the training dataset to simulate the online bandit with 10 arms corresponding to different digits. For STL-10, 100,000 samples of unlabeled data are used to pre-train the encodings; then the 5,000 test samples together with 8,000 training samples are combined to simulate the online bandit, again with 10 different arms corresponding to image classes ${ }^{2}$ For Caltech-101 Silhouettes-28 dataset, out of the original 8671 samples, 671 are used for pre-training and 8000 for online learning with 101 different arms (class labels). For CIFAR-10 dataset, 10,000 test set samples are used for pre-training, and 50,000 training samples are left for the online bandit with 10 arms (classes). For Warfarin dataset, 528 test set samples are used for pre-training, and 5,000 training samples are left for the online bandit with 3 arms (classes).

\section{B. Nonstationary Environments}

We simulated several types of nonstationarity using the above datasets (as in [Lin, 2020a]). As mentioned before, we assume that the input data arrive in batches, and the data distribution (i.e., the joint distribution of the context and reward) may change across those batches, while remaining stationary within each batch. We used the batch size of 1,000, and varied the number of embeddings $k$, using $k=2$, 4 , or 8 , presenting average results over all $k$.

1) Nonstationary context: varying cluster distribution: To simulate changes in the context (input) distribution, we first clustered all samples in the corresponding pre-training data subset into $k$ clusters. Next, we generate a sequence of batches, where each batch contained a certain fraction of samples from different clusters, and these fractions were changing across the batches, i.e. the probability distribution of cluster membership was changing, simulating nonstationary input.

2) Nonstationary context: negative images: Another type of input nonstationarity involved introducing negative images as inputs with same semantics but different textures. Namely, with probability $p$, the negative image of the original image was presented as an input. Experiments were performed in two settings: half $(p=0.5)$ and rand $(0<p<1$ randomly assigned for each mini-batch), in both stationary and nonstationary context conditions, with both shuffled and unshuffled rewards (described later).

3) Nonstationary reward: multi-task environment: Another type of nonstationarity was assuming that input samples may come from different domains (tasks), and thus can be associated

\footnotetext{
${ }^{2}$ To speed up the computation, we squeezed input 27648-dimensional vectors into 1000-dimensional ones via linear stretching.
} 
with different subsets of labels (arms). For example, we combined 5,000 randomly selected training samples from each of the two selected domains, MNIST and Warfarin datasets, and extended the set of possible labels (arms) to include 10 labels from MNIST and 3 labels from Warfarin. We used linear stretching to make the input dimensions equal across the two domains. The algorithm had to assign a label to each input without any information about which domain the input came from.

4) Nonstationary reward: shuffled class labels: We further explored the multi-task setting by introducing a different type of nonstationary reward, where the class labels were shuffled, i.e. randomly permuted, in each batch.

\section{Results}

We explored different combination of the above nonstationarities. Table II summarizes our results for the nonstationary context due to varying cluster distribution, and for mixeddomain (multi-task) settings, with unshuffled reward function. As we can see, on three out of six datasets, baseline was still outperforming our embeddings. However, if we consider the mean accuracy in the entire set of experiments, the top three algorithms were: universal embedding (mean accuracy 28.83\%), baseline (mean accuracy 27.78\%), mini-batch embedding (mean accuracy $27.58 \%$ ), respectively, suggesting the advantage of representation learning (embedding computation). Moreover, if we take a look at the whole iteration history, for example, for MNIST dataset (Figure 1), we observe that initially, the baseline CB (solid line) is considerably worse than embedding-based approaches, and requires a large number of iteration to finally catch up with them. Figures 2 and 3 show the history of reward accumulation for the STL-10 and CIFAR-10, demonstrating that the baseline is consistently outperformed by embedding selection methods.

TABLE II: Nonstationary Environment with Unshuffled Labels

\begin{tabular}{l|l|l|l|l} 
Datasets & baseline & $\mathrm{uE}$ & $\mathrm{mE}$ & $\mathrm{oE}$ \\
\hline MNIST & $\mathbf{3 7 . 2 4}$ & 34.44 & 29.00 & 22.32 \\
STL-10 & 10.29 & $\mathbf{1 5 . 8 1}$ & 14.77 & 13.43 \\
CIFAR-10 & 9.62 & $\mathbf{1 4 . 3 0}$ & 13.30 & 11.73 \\
Caltech-101 S & $\mathbf{1 . 1 8}$ & 1.14 & 1.09 & 1.06 \\
Warfarin & $\mathbf{6 2 . 5 8}$ & 56.70 & 56.10 & 56.92 \\
\hline mix: MNIST/Warfarin & 45.76 & 50.58 & $\mathbf{5 1 . 2 1}$ & 47.74 \\
\hline
\end{tabular}

TABLE III: Nonstationary Environment with Shuffled Labels

\begin{tabular}{l|l|l|l|l} 
Datasets & baseline & $\mathrm{uE}$ & $\mathrm{mE}$ & $\mathrm{oE}$ \\
\hline MNIST & 12.19 & $\mathbf{3 3 . 7 5}$ & 29.04 & 23.83 \\
STL-10 & 10.05 & $\mathbf{1 6 . 6 4}$ & 15.10 & 12.77 \\
CIFAR-10 & 10.23 & $\mathbf{1 4 . 8 3}$ & 13.13 & 11.60 \\
Caltech-101 S & 1.00 & 1.09 & 1.23 & $\mathbf{1 . 3 0}$ \\
Warfarin & 40.66 & $\mathbf{5 5 . 1 0}$ & 50.56 & 54.44 \\
\hline mix: MNIST/Warfarin & 23.54 & 49.33 & $\mathbf{5 0 . 6 7}$ & 49.15 \\
\hline
\end{tabular}

Next, Table III summarizes our results with shuffled reward function, for the nonstationary context due to varying cluster distribution, and for mixed-domain (multi-task) settings. Based on the mean accuracy in the entire experiment, the top three algorithms were: universal embedding (mean accuracy $28.46 \%$ ),
TABLE IV: Negative Environment with Unshuffled Labels

\begin{tabular}{l|l|l|l|l} 
Datasets & baseline & $\mathrm{uE}$ & $\mathrm{mE}$ & $\mathrm{oE}$ \\
\hline MNIST half-stat & 13.50 & 14.70 & 14.02 & $\mathbf{1 6 . 1 8}$ \\
MNIST rand-stat & 13.72 & 17.14 & 15.53 & $\mathbf{1 7 . 7 0}$ \\
MNIST half-nonStat & 14.45 & 25.09 & 23.82 & $\mathbf{2 6 . 9 0}$ \\
MNIST rand-nonStat & 14.05 & 24.38 & 25.90 & $\mathbf{2 8 . 4 3}$ \\
\hline STL-10 half-stat & 10.06 & $\mathbf{1 0 . 4 2}$ & 10.33 & 10.04 \\
STL-10 rand-stat & 9.77 & $\mathbf{1 2 . 3 4}$ & 12.33 & 10.41 \\
STL-10 half-nonStat & 9.88 & 10.99 & $\mathbf{1 2 . 2 9}$ & 11.56 \\
STL-10 rand-nonStat & 9.85 & 12.99 & $\mathbf{1 3 . 6 7}$ & 11.55 \\
\hline Caltech-101 S half-stat & 0.98 & $\mathbf{1 0 . 0 4}$ & 7.98 & 6.94 \\
Caltech-101 S rand-stat & 0.94 & 10.93 & 8.40 & $\mathbf{1 1 . 6 8}$ \\
Caltech-101 S half-nonStat & 1.04 & 1.20 & $\mathbf{1 . 2 3}$ & 0.96 \\
Caltech-101 S rand-nonStat & 0.96 & 1.09 & $\mathbf{1 . 2 0}$ & 0.99 \\
\hline
\end{tabular}

TABLE V: Negative Environment with Shuffled Labels

\begin{tabular}{l|l|l|l|l} 
Datasets & baseline & $\mathrm{uE}$ & $\mathrm{mE}$ & $\mathrm{oE}$ \\
\hline MNIST half-stat & 10.22 & 14.59 & 13.86 & $\mathbf{1 4 . 7 9}$ \\
MNIST rand-stat & 9.87 & $\mathbf{1 7 . 8 4}$ & 14.35 & 17.32 \\
MNIST half-nonStat & 10.78 & 23.02 & 22.33 & $\mathbf{2 6 . 8 4}$ \\
MNIST rand-nonStat & 11.27 & 27.34 & 24.87 & $\mathbf{2 8 . 3 6}$ \\
\hline STL-10 half-stat & 9.66 & $\mathbf{1 1 . 5 1}$ & 10.73 & 10.60 \\
STL-10 rand-stat & 9.95 & 11.44 & $\mathbf{1 2 . 3 7}$ & 11.17 \\
STL-10 half-nonStat & 10.31 & 11.86 & $\mathbf{1 3 . 1 7}$ & 11.19 \\
STL-10 rand-nonStat & 9.2 & $\mathbf{1 2 . 6 2}$ & 12.49 & 11.59 \\
\hline Caltech-101 S half-stat & 1.11 & $\mathbf{8 . 7 1}$ & 6.21 & 7.93 \\
Caltech-101 S rand-stat & 0.94 & $\mathbf{1 0 . 3 6}$ & 9.11 & 3.38 \\
Caltech-101 S half-nonStat & 1.06 & 1.03 & 1.00 & $\mathbf{1 . 2 9}$ \\
Caltech-101 S rand-nonStat & 1.08 & 1.05 & 1.13 & $\mathbf{1 . 1 6}$ \\
\hline
\end{tabular}

mini-batch embedding (mean accuracy 26.62\%), online embedding (mean accuracy $25.52 \%$ ), respectively. Furthermore, in this experiment, our embedding-based approaches always outperformed the baseline, suggesting that in a setting where reward functions are nonstationary, in addition to the nonstationary input environment, the advantage of representation learning is quite significant, as compared to standard CB (mean accuracy $16.28 \%$ ). Note that, with nonstationary (shuffled) labels, the reward accumulated by the baseline $\mathrm{CB}$ remains significantly below the reward of embedding-based approaches, at all iterations (Figures 4,6). Thus, in a more challenging setting with both context and reward nonstationarities, the embedding-based approaches clearly outperform the standard contextual bandit.

Table IV summarizes our results for the nonstationary online learning setting with negative environments and unshuffled reward. Based on the mean accuracy in the entire experiment, the top three algorithms were: online embedding (mean accuracy $12.78 \%$ ), universal embedding (mean accuracy 12.61\%), minibatch embedding (mean accuracy 12.23\%), respectively. Again, the embedding-based approaches are always superior to the baseline $\mathrm{CB}$; online embedding achieved the best performance among all methods on MNIST, while universal and batch embeddings were taking their turns outperforming the baseline on other datasets and settings.

Finally, Table $\mathrm{V}$ summarizes our results for the nonstationary online learning setting with negative environments and shuffled reward function. Based on the mean accuracy in the entire experiment, the top three algorithms were: universal embedding (mean accuracy 12.61\%), online embedding (mean accuracy $12.14 \%$ ), mini-batch embedding (mean accuracy $11.80 \%$ ), 


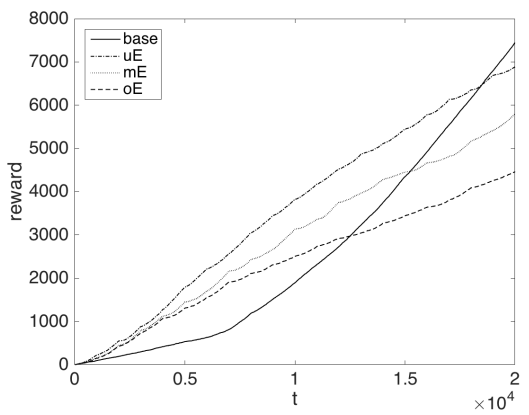

Fig. 1: MNIST unshuffled, $\mathrm{k}=2$

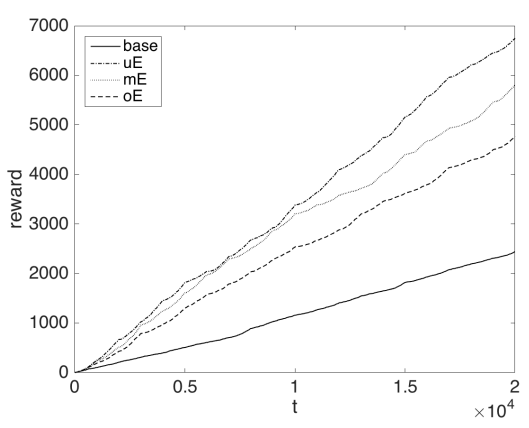

Fig. 4: MNIST shuffled, $\mathrm{k}=2$

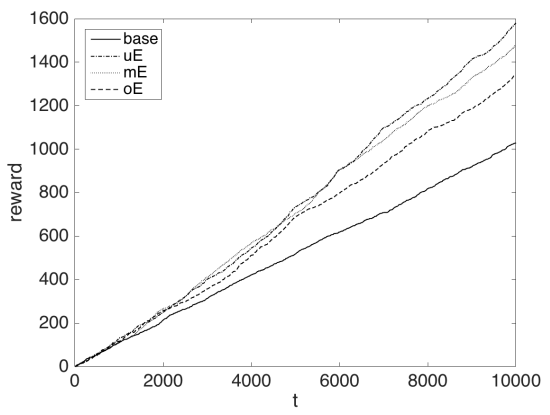

Fig. 2: STL-10 unshuffled, $\mathrm{k}=2$

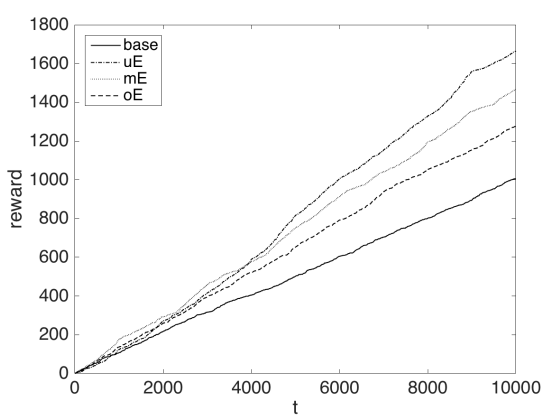

Fig. 5: STL-10 shuffled, $k=2$

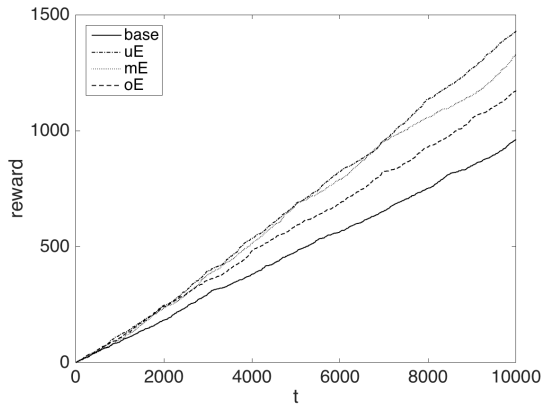

Fig. 3: CIFAR-10 unshuffled, $\mathrm{k}=2$

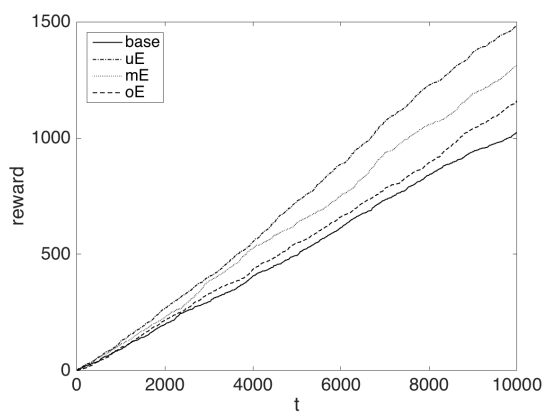

Fig. 6: CIFAR-10 shuffled, $\mathrm{k}=2$

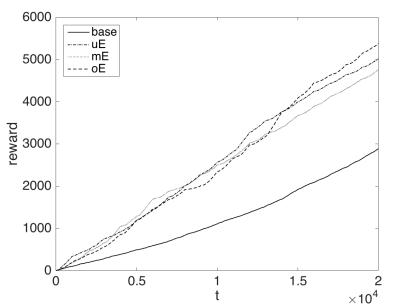

Fig. 7: MNIST unshuffled halfnonStat

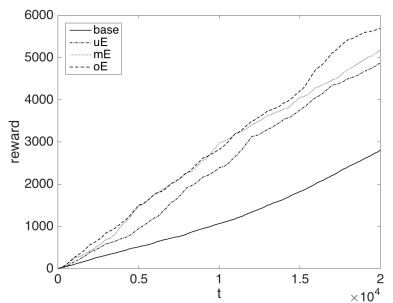

Fig. 8: MNIST unshuffled randnonStat

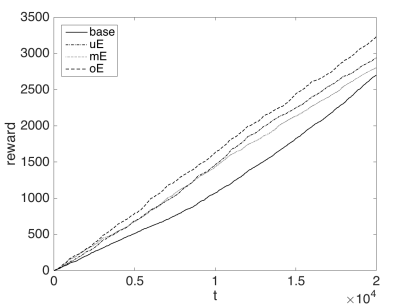

Fig. 9: MNIST unshuffled halfstat

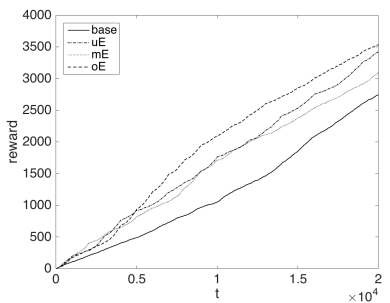

Fig. 10: MNIST unshuffled randstat respectively, further confirming the advantage of adaptive encoding over standard CB (mean accuracy $7.12 \%$ ). In addition, the difference of textures under the same semantics introduced in this experiments demonstrated that embedding selection outperforms single universal embedding in most nonstationary cases.

Figures 7, 14 visualize the details of reward accumulation over time by different methods, on MNIST data and all the settings from the Tables [V] and $\mathrm{V}$. The performance gap between the embedding-based approaches and the baseline is especially large in those settings. Furthermore, we can see that both adaptive, context-dependent embedding approaches ( $\mathrm{EE}$ and $\mathrm{mE}$ ) consistently ourperform the single-embedding approach $(\mathrm{uE})$, with the online embedding emerging as the best one, especially with increasing number of iterations.

\section{CONCLUSIONS}

We introduced an extension of the contextual bandit problem motivated by several real-world applications in non-stationary environments, including recommendation systems, health monitoring and medical diagnosis, and others. In this setting, which we refer to as Contextual Bandit with Representation learning and unlabeled History (CBRH), a set of unlabeled contexts is available prior to online decision making, which allows, instead of using the raw context, to learn context representations. Next, during the online phase, embeddings are selected adaptively, depending on each context, and updated based on the contexts observed so far. We propose two specific algorithms for the CBRH problem, based on online and offline clustering, which combine online embedding selection and learning with contextual Thompson Sampling bandit. The 


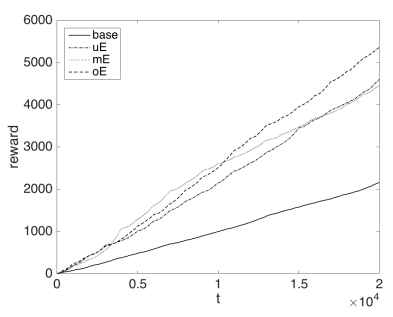

Fig. 11: MNIST shuffled halfnonStat

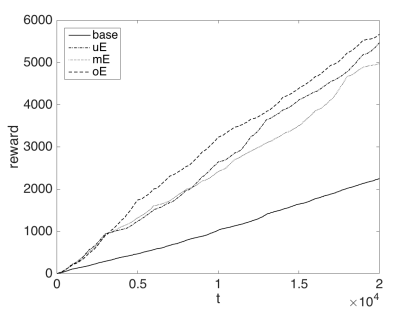

Fig. 12: MNIST shuffled randnonStat

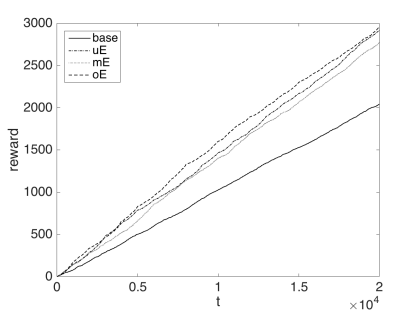

Fig. 13: MNIST shuffled half-stat

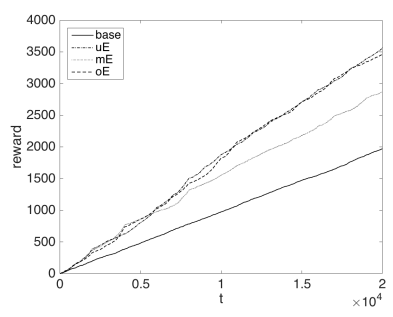

Fig. 14: MNIST shuffled randstat algorithms are evaluated in several types of nonstationary environments and compared to the standard contextual bandit, as well as universal (single) embedding, on several datasets. Overall, we observe clear advantages of the embedding-based approaches over the standard contextual bandit; moreover, the proposed adaptive embedding selection and learning methods frequently outperform the universal embedding in multiple nonstationary settings.

\section{REFERENCES}

Shipra Agrawal and Navin Goyal. Analysis of thompson sampling for the multi-armed bandit problem. In COLT 2012 - The 25th Annual Conference on Learning Theory, June 25-27, 2012, Edinburgh, Scotland, pages 39.1-39.26, 2012.

Shipra Agrawal and Navin Goyal. Thompson sampling for contextual bandits with linear payoffs. In ICML (3), pages 127-135, 2013.

Robin Allesiardo, Raphaël Féraud, and Djallel Bouneffouf. A neural networks committee for the contextual bandit problem. In Neural Information Processing - 21st International Conference, ICONIP 2014, Kuching, Malaysia, November 3-6, 2014. Proceedings, Part I, pages 374-381, 2014.

Peter Auer and Nicolò Cesa-Bianchi. On-line learning with malicious noise and the closure algorithm. Ann. Math. Artif. Intell., 23(1-2):83-99, 1998.

Peter Auer, Nicolò Cesa-Bianchi, and Paul Fischer. Finitetime analysis of the multiarmed bandit problem. Machine Learning, 47(2-3):235-256, 2002.

Peter Auer, Nicolò Cesa-Bianchi, Yoav Freund, and Robert E. Schapire. The nonstochastic multiarmed bandit problem. SIAM J. Comput., 32(1):48-77, 2002.

Gábor Bartók, Dean P Foster, Dávid Pál, Alexander Rakhlin, and Csaba Szepesvári. Partial monitoring-classification, regret bounds, and algorithms. Mathematics of Operations Research, 39(4):967-997, 2014.

Djallel Bouneffouf and Raphaël Féraud. Multi-armed bandit problem with known trend. Neurocomputing, 205:16-21, 2016.

Djallel Bouneffouf, Irina Rish, and Guillermo A Cecchi. Bandit models of human behavior: Reward processing in mental disorders. In International Conference on Artificial General Intelligence, pages 237-248. Springer, 2017.
Djallel Bouneffouf, Irina Rish, Guillermo A Cecchi, and Raphaël Féraud. Context attentive bandits: Contextual bandit with restricted context. In Proceedings of IJCAI-2017, 2017.

Wei Chu, Lihong Li, Lev Reyzin, and Robert E. Schapire. Contextual bandits with linear payoff functions. In Geoffrey J. Gordon, David B. Dunson, and Miroslav Dudik, editors, AISTATS, volume 15 of JMLR Proceedings, pages 208-214. JMLR.org, 2011.

Adam Coates and Andrew Y Ng. The importance of encoding versus training with sparse coding and vector quantization. In Proceedings of the 28th International Conference on Machine Learning (ICML-11), pages 921-928, 2011.

Adam Coates, Andrew Ng, and Honglak Lee. An analysis of single-layer networks in unsupervised feature learning. In Proceedings of the fourteenth international conference on artificial intelligence and statistics, pages 215-223, 2011.

International Warfarin Pharmacogenetics Consortium et al. Estimation of the warfarin dose with clinical and pharmacogenetic data. N Engl J Med, 2009(360):753-764, 2009.

Pratik Gajane, Tanguy Urvoy, and Emilie Kaufmann. Corrupt bandits. EWRL, 2016.

Gregory Griffin, Alex Holub, and Pietro Perona. Caltech-256 object category dataset. 2007.

T. L. Lai and Herbert Robbins. Asymptotically efficient adaptive allocation rules. Advances in Applied Mathematics, 6(1):4-22, 1985.

John Langford and Tong Zhang. The epoch-greedy algorithm for multi-armed bandits with side information. In Advances in neural information processing systems, pages 817-824, 2008.

Yann LeCun. The mnist database of handwritten digits. http://yann. lecun. com/exdb/mnist/, 1998.

Lihong Li, Wei Chu, John Langford, and Robert E Schapire. A contextual-bandit approach to personalized news article recommendation. In Proceedings of the 19th International Conference on World Wide Web (WWW2010), pages 661-670. ACM, 2010.

Baihan Lin and Xinxin Zhang. Speaker diarization as a fully online learning problem in minivox. arXiv preprint arXiv:2006.04376, 2020.

Baihan Lin and Xinxin Zhang. VoiceID on the fly: A speaker recognition system that learns from scratch. In INTERSPEECH, 2020. 
Baihan Lin, Djallel Bouneffouf, and Guillermo Cecchi. Split Q learning: Reinforcement learning with two-stream rewards. In Proceedings of the Twenty-Eighth International Joint Conference on Artificial Intelligence, IJCAI-19, pages 6448-6449. International Joint Conferences on Artificial Intelligence Organization, 72019.

Baihan Lin, Djallel Bouneffouf, and Guillermo Cecchi. Online learning in iterated prisoner's dilemma to mimic human behavior. arXiv preprint arXiv:2006.06580, 2020.

Baihan Lin, Guillermo Cecchi, Djallel Bouneffouf, Jenna Reinen, and Irina Rish. A story of two streams: Reinforcement learning models from human behavior and neuropsychiatry. In Proceedings of the Nineteenth International Conference on Autonomous Agents and Multi-Agent Systems, AAMAS-20, pages 744-752. International Foundation for Autonomous Agents and Multiagent Systems, 52020.

Baihan Lin, Guillermo Cecchi, Djallel Bouneffouf, Jenna Reinen, and Irina Rish. Unified models of human behavioral agents in bandits, contextual bandits and rl. arXiv preprint arXiv:2005.04544, 2020.

Baihan Lin. Diabolical games: Reinforcement learning environments for lifelong learning. under review, 2020.

Baihan Lin. Online semi-supervised learning in contextual bandits with episodic reward. In Australasian Joint Conference on Artificial Intelligence. Springer, 2020.

Jérémie Mary, Romaric Gaudel, and Philippe Preux. Bandits and recommender systems. In Machine Learning, Optimization, and Big Data - First International Workshop, MOD 2015, pages 325-336, 2015.

II Ororbia, G Alexander, C Lee Giles, and David Reitter. Online semi-supervised learning with deep hybrid boltzmann machines and denoising autoencoders. arXiv preprint arXiv:1511.06964, 2015.

W.R. Thompson. On the likelihood that one unknown probability exceeds another in view of the evidence of two samples. Biometrika, 25:285-294, 1933.

Sofía S Villar, Jack Bowden, and James Wason. Multi-armed bandit models for the optimal design of clinical trials: benefits and challenges. Statistical science: a review journal of the Institute of Mathematical Statistics, 30(2):199, 2015.

B. Yver. Online semi-supervised learning: Application to dynamic learning from radar data. In 2009 International Radar Conference "Surveillance for a Safer World" (RADAR 2009), pages 1-6, Oct 2009. 